\title{
Prediction of blood pressure from plasma renin activity in reflux nephropathy
}

\author{
H Jardim, V Shah, J M Savage, T M Barratt, M J Dillon
}

\begin{abstract}
As there is a $10 \%$ risk of hypertension developing in children with reflux nephropathy and the renin-angiotensin system has been implicated in its aetiology, a long term prospective study has been undertaken to explore the relationship between plasma renin activity (PRA) and blood pressure in such patients. In 1978, of 100 normotensive children with reflux nephropathy $8 \%$ were shown to have PRA above normal. Five years later of 85 subjects suitable for analysis $13 \%$ had increased PRA and it was shown that PRA and blood pressure SD scores significantly increased. The present study refers to the $\mathbf{1 0}$ year follow up in which 95 of the original group were traced but eight of these were unavailable for study and 28 others were excluded from analysis because of extraneous factors that might influence blood pressure or PRA. Results therefore on 59 have been analysed. PRA was above normal in $13 / 59$ (20\%) subjects, and PRA and blood pressure SD scores had further increased. The data continue to support the role of the renin-angiotensin system in the observed rise of blood pressure in reflux nephropathy, but individual PRA measurements do not appear so far to predict reliably the onset of hypertension in affected patients.
\end{abstract}

The commonest cause of severe hypertension in childhood is pyelonephritic scarring secondary to urinary tract infection and vesicoureteric reflux. ${ }^{1-3}$ Hypertension affects at least $10 \%$ of these patients and, once present, hypertension can accelerate the deterioration of renal function. ${ }^{4-7}$ Furthermore, hypertension is associated with considerable morbidity and mortality, which makes early diagnosis and treatment extremely important. ${ }^{8-10}$ The aetiology of the hypertension in these patients is not clear. The renin-angiotensin system has been implicated due to the frequent finding of a raised plasma renin activity (PRA) in hypertensive patients with reflux nephropathy. ${ }^{11}$

In an attempt to identify the role of PRA as a possible predictor of the development of hypertension in these patients, in 1978 we started a long term prospective study in 100 normotensive children known to have renal scars. ${ }^{12}$ At that time $8 / 100$ patients had increased PRA and the natural tendency of PRA to fall with age was less pronounced than in normal controls. ${ }^{12}$ Five years later 98 of the initial 100 were reviewed and a significant increase in blood pressure and PRA SD scores was found suggesting that there was a relationship between renin and blood pressure in these subjects. ${ }^{13}$ The present study concerns the 10 year follow up of this population.

\section{Patients and methods}

One hundred children with radiologically identifiable renal scarring and vesicoureteric reflux that had been corrected surgically were studied in 1978. At the five year review, 98 of the original 100 patients were contacted. One patient could not be traced and the other had died with hypertensive encephalopathy. Although all 98 patients were investigated, a number were unsuitable for analysis. Thirteen patients were excluded: 11 because they were taking a hormonal contraceptive pill, one because she was pregnant, and another because she had undergone a nephrectomy.

For the 10 year review we traced 95 patients from the original group. However, only 87 patients were studied due to various factors: three refused to cooperate, four were living abroad, and one was neurologically handicapped after a car accident. Of the 87 reviewed, data on 28 were excluded, in 24 because they were taking a hormonal contraceptive pill and in four because they had had a nephrectomy because of hypertension. Data on 59 patients were therefore analysed. There were 30 male and 29 female patients aged 11-25 (mean 17.4) years. None of the patients were receiving antihypertensive treatment or known to have impaired renal function.

Due to their age most of the patients were no longer under the care of the Hospital for Sick Children and were specifically invited to take part in this third follow up study.

Each patient was asked to attend the Hospital for Sick Children in the morning. After a two hour supine rest, blood pressure was measured with a Dinamap vital signs monitor and with a random zero sphygmomanometer. The observer was always the same. A $1 \mathrm{ml}$ blood sample was taken for measurement of electrolyte and creatinine concentrations and for PRA estimation. PRA was evaluated using a semimicroradioimmunoassay ${ }^{14}$ and plasma creatinine concentration by the Jaffé reaction with an autoanalyser.

A urine sample was also obtained and mean sodium excretion calculated from the sodium: creatinine ratio assuming that normal daily creatinine excretion is constant at $132 \mathrm{mmol} / \mathrm{kg}$ body weight/24 hours. ${ }^{12} 15$

Blood pressure results from the three studies were expressed as SD scores standardised against the 1987 Task Force data for blood pressure in normal children ${ }^{16}$ and calculated using a computer program. 
Plasma renin activity SD scores were calculated in order to compare the results from the three evaluations. Renin results were logarithmically transformed and SD scores calculated according to the equation:

$$
\text { SD score }=\frac{\log \text { PRA }- \text { mean } \log \text { PRA }}{\log S D}
$$

Where log PRA= log PRA observed, mean log $\mathrm{PRA}=$ mean log $\mathrm{PRA}$ for age, and $\log \mathrm{SD}=\log$ standard deviation.

The results on PRA and blood pressure SD scores from the first to the second and from the second to the third studies were compared by paired Student's $t$ test.

The degree of renal scarring was assessed from the most recent scanning with dimercaptosuccinic acid labelled with technetium-99m available. This was scored as in the original study: normal kidney $=0$, single small polar scar $=1$, complete polar destruction $=2$, and loss of both renal poles or a small shrunken kidney= 3. It was not possible to obtain recent renal imaging in all the patients to reassess the degree of scarring, but the patients in whom this was obtained showed no change from the second study.

\section{Results}

In the first study in 1978, eight of the 100 patients $(8 \%)$ had a PRA above the upper limit of normal for age. ${ }^{12}$ Five years later, 11 of the 85 patients $(13 \%)$ studied showed a raised PRA. ${ }^{13}$ In the present study PRA was above the normal range in 12 patients $(20 \%)$ of the 59 studied (fig 1). Four of these patients had a blood pressure

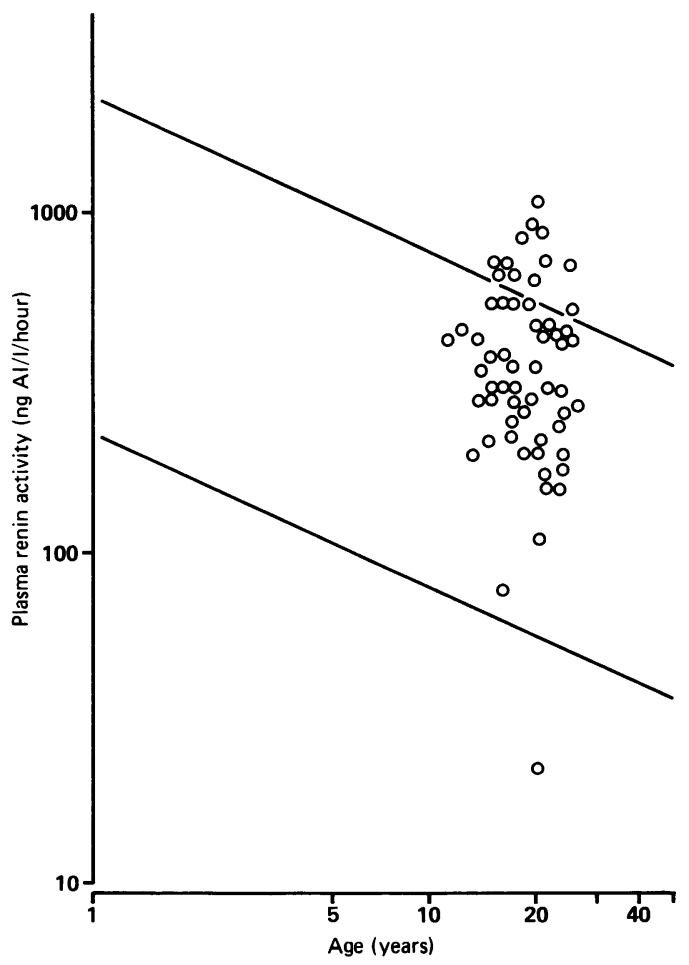

Figure 1 Plasmareninactivity in normotensivepatients with scarred kidneys in 1988. Solid lines indicate the upper and lower limits of the normal range. above the 95th centile and two of them had had a raised PRA in the second study although normal values in 1978. Only three patients showed a high PRA in all studies and only one became significantly hypertensive at the present review.

As in the previous investigations, PRA SD scores were calculated so that we could compare renin results from the various studies. The mean (SE) PRA SD score in 1978 was 0.042 $(0 \cdot 14)$, in the second study $0.453(0.13)$, and in this final evaluation $0 \cdot 845(0 \cdot 14)$. There was a highly significant increase in PRA SD scores between the first and the second studies $(p<0.001)$ and a significant increase between the second and the third $(p<0.05)$ (fig 2$)$. The patient with a PRA of -3 SD scores below the mean (fig 2) was one of the oldest and had only a minor degree of unilateral scarring (grade 1).

In this third follow up study no significant correlation could be found between PRA and mean sodium excretion, plasma creatinine concentration, or the degree of scarring. Similar results were found before. ${ }^{12} 13$

All patients were originally normotensive when first studied in $1978 .^{12}$ Five years later blood pressure was above the 95th centile in three patients and between the 90-95th centiles in four. ${ }^{13}$ Only one of these seven patients had a high PRA at that time. ${ }^{13}$ Of the original group of 100 patients, one had undergone unilateral nephrectomy due to hypertension at the time of the second study, however, his original PRA determination was within the normal range for age. The group of three patients identified as having hypertension in the second study had all been submitted to unilateral nephrectomies at the latest review. As with the patient who was nephrectomised between the two initial studies, the PRA results were within the normal range five and also 10 years earlier. Although not included in the present analysis, two of these patients remained hypertensive when last

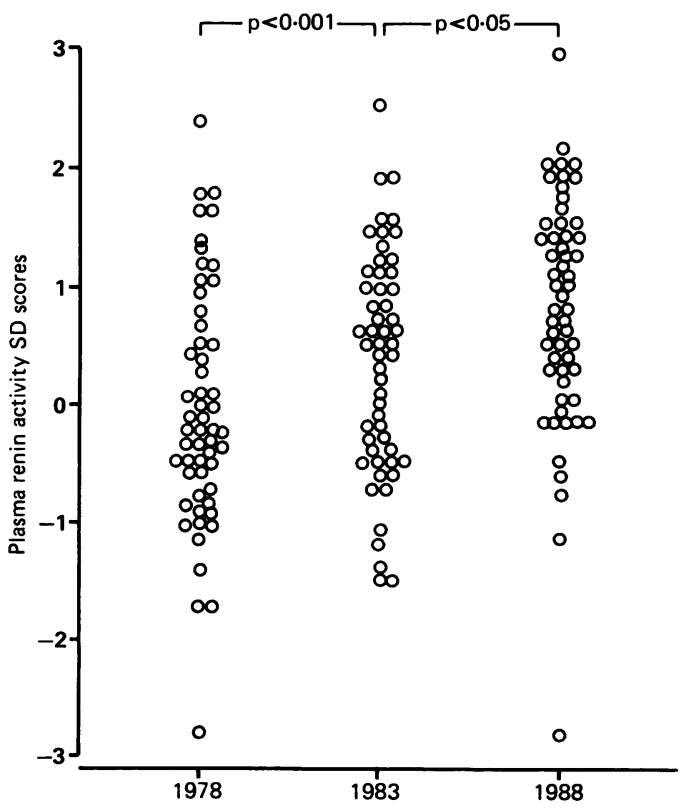

Figure 2 Plasma reninactivity SD scores from the initial, five, and 10 year follow up studies. 
reviewed, and one had a high PRA while on treatment with a $\beta$ blocker.

In the present analysis we identified a further eight patients with a blood pressure over the 95th centile and seven with a blood pressure between the 90-95th centiles. However, in only six of these 15 patients was PRA above the normal for age.

As for PRA, SD scores on blood pressure results were calculated in order to be compared. The initial mean (SE) blood pressure SD score was $-0.26(0.22)$, five years later $0.49(0.11)$, and $0.97(0.12)$ in this present study. There was a highly significant increase in systolic blood pressure SD score from the first to the second study ( $p<0.001)$ as well as between the second and third ( $p<0.001$ ) (fig 3 ). A similar observation was found for diastolic blood pressure SD score.

No significant correlation was found between blood pressure and PRA SD scores. We were also unable to find a significant correlation between the variation in blood pressure and PRA SD scores either between the first and second or between the second and third studies.

\section{Discussion}

Hypertension is a frequent complication of reflux nephropathy. ${ }^{3-5}$ PRA is often increased in hypertensive patients with this condition. ${ }^{11}$ When present in these patients, hypertension contributes to further deterioration of renal function. ${ }^{7}$ Therefore an early marker of its onset would be useful in the identification of patients at risk of developing a high blood pressure.

In an attempt to clarify the relationship between blood pressure and PRA in patients with reflux nephropathy, we undertook a long term prospective study. The initial results obtained in 1978 showed that eight of 100 normotensive patients had a raised PRA and we

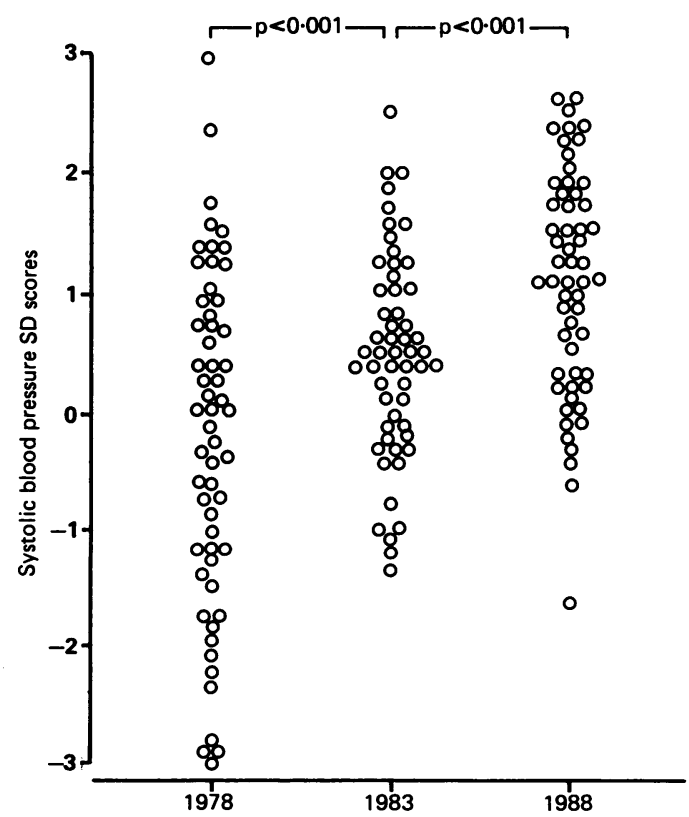

Figure 3 Systolic blood pressure SD scores from the initial, five, and 10 year follow up studies. then postulated that renin could predate, and then predict, hypertension. ${ }^{12}$ Five years later only one of the eight patients identified with a blood pressure above the 90th centile had had a raised PRA five years earlier. ${ }^{13}$ Ten years later six of the 15 patients with a blood pressure above the 90th centile showed a high PRA. Furthermore only two of these had an increased PRA in the second review and only one patient with raised PRA in the three studies became significantly hypertensive at the last review. The present results and those from the preceding study do not confirm that isolated PRA estimations can reliably predict the onset of hypertension in patients with reflux nephropathy.

On the other hand the comparison of SD scores for PRA between the first and the last study show a significant increase during the 10 year duration of the study implying that there is a disturbance of the renin-angiotensin system in these patients. Blood pressure SD scores were also significantly increased which confirms that in reflux nephropathy there is a tendency towards high blood pressure and that this may be a delayed event in life.

Interestingly, the three patients identified as hypertensive (with blood pressure $>95$ th centile) in the second study, had normal PRA at that stage as well as in the first evaluation but subsequently required unilateral nephrectomies due to severe hypertension. This could be taken as an example of the difficulty in predicting the hypertension development based on renin measurements.

The number of patients taking hormonal contraceptive pills was considerably higher in this third study and led to the exclusion of data from 24 patients from analysis, due to the problems in renin results interpretation in the patients taking these drugs. ${ }^{17}$ Of these 24 girls, six had a high PRA and two of these had a blood pressure over the 95th centile for age. Blood pressure was found to be raised also in six girls with normal PRA who were taking the contraceptive pill.

This third study of the same population with reflux nephropathy over a 10 year period continues to support the view that the reninangiotensin system is abnormal in patients with reflux nephropathy and that this is accompanied by an abnormally high tendency towards high blood pressure. However, individual PRA measurements do not appear, so far, to predict reliably the onset of hypertension in affected patients.

The role of renin as the only determinant of hypertension in reflux nephropathy remains to be established. The study of other factors with a possible role in the aetiology of hypertension in this condition is planned at subsequent follow up studies of this population.

HJ was supported by Junta Nacional de Investigacao Cientifica e Tecnologica, Lisbon, Portugal and VS by the Kidney Research Aid Fund. 
2 Gill DG, De Costa BM, Cameron JS, Joseph MC, Ogg CS, Chantler C. Analysis of 100 children with severe and persistent hypertension. Arch Dis Child 1976;51:951-6.

3 Dillon MJ. Clinical aspects of hypertension. In: Holliday MA, Barratt TM, Vernier RL, eds. Pediatric nephrology. 2nd Ed. Baltimore: Williams and Wilkins, 1987:743-57.

4 Wallace DMA, Rothwell DL, Williams DI. The long term follow-up of surgically treated vesico-ureteric reflux. $\mathrm{Br}$ F Urol 1978;50:479-84.

5 Gusmano R, Perfumo F, Raspino M, Ginevri F, Verrina E, Ciardi MR. Natural history of reflux nephropathy in children. Contrib Nephrol 1988;61:201-9.

6 Smellie JM, Normand ICS. Reflux nephropathy in childhood. In: Hodson CJ, Kincaid-Smith P, eds. Reflux nephropood. In: Hodson CJ, Kincaid-Smith P, eds. Reflux

7 Lathy. New York: Mason Publishing, 1979:14-20. blood pressure and the rate of decline in renal function with blood pressure and the rate of
age. Kidney Int 1984;26:861-8.

8 Bailey RR. Long term follow-up of infants with gross vesicoureteric reflux. In: Hodson CJ, Heptinstall RH, Winberg J, eds. Contributions to nephrology. Reflux nephropathy, update, 1983. Basle: Karger, 1984:146-51.

9 Braren V, West JC, Boerth RC, Harmon CM. Management of children with hypertension from reflux or obstructive nephropathy. Urology 1988;32:228-34.

10 Smellie JM, Daman Willems CE. Vesico-ureteric reflux: recent research and its effect upon clinical practice. In:
Catto GRD, ed. Urinary tract infection. Dordrecht: Kluwer Academic Publishers, 1989:39-85.

11 Dillon MJ, Smellie JM. Peripheral plasma renin activity, hypertension and renal scarring in children. In: Hodson CJ, Heptinstall RH, Winberg J, eds. Contributions to nephrology. Reflux nephropathy update, 1983. Basle: Karger, 1984:68-80.

12 Savage JM, Dillon MJ, Shah V, Barratt TM, Williams DI. Renin and blood pressure in children with renal scarring and vesicoureteric reflux. Lancet 1978;ii:441-4.

13 Savage JM, Koh CT, Shah V, Barratt TM, Dillon MJ. Five year prospective study of plasma renin activity and blood pressure in patients with long standing reflux nephropathy. Arch Dis Child 1987;62:678-82.

14 Dillon MJ. Measurement of plasma renin activity by semimicro radioimmunoassay of generated angiotensin $\mathrm{I}$. $\mathcal{f} \mathrm{Clin}$ Pathol 1975;28:625-30.

15 Graystone JE. Creatinine excretion during growth. In: Cheek DB, ed. Human growth. Philadelphia: Lea and Febiger, 1968:182-97.

16 National Heart, Lung and Blood Institute, Bethesda, Maryland. Report of the second task force on blood pressure control in children, 1987. Pediatrics 1987;79:1-25.

17 Kotchen TA, Kotchen JM, Guthrie GP, Cottrill CM. Plasma renin activity, reactivity, concentration and substrate following hypertension during pregnancy. Effect of ora contraceptive agents. Hypertension 1979;1:355-61.

Group A streptococcal exotoxins

When I wrote recently about the reported resurgence of group A streptococcal disease in the United States, ${ }^{1}$ I didn't realise that a similar increase had already been described in Britain. In the discussion section of a paper in the Pediatric Infectious Disease Fournal (Kiran Belani and colleagues, 1991;10:351-4) it is pointed out that in England and Wales the annual death rate from group A streptococcal septicaemia rose from between five and 10 in the early 1980 s to between 15 and 22 by the end of the decade. There were about 150 cases a year of group A streptococcal bacteraemia in the second half of the 1970s and 300 a year in the first half of the 1980s. This paper, from Minneapolis, suggests that a change in exotoxin production by the bacteria may be behind the resurgence of severe disease. There are three pyrogenic exotoxins (A, B, and $C$ ) produced by group A streptococci, toxin A being the classical erythrogenic toxin of scarlet fever. Toxin A production was common 50 years ago in the heyday of scarlet fever but has been uncommon in recent decades. In the hospital of the University of Minnesota, Minneapolis, six children with severe group A streptococcal disease were seen between 1985 and 1988. One was a 2 week old baby who probably acquired the organism from a sibling with pharyngitis. The other five were aged 3 to 14 years and all had a predisposing condition: two had congenital lymphatic abnormalities, one a recent arm fracture, one eczema, and one chickenpox. (Apparently it is known that severe streptococcal disease may follow chickenpox.) The principal features seen in the six cases were cellulitis, disseminated intravascular coagulation, shock, and gangrene leading to limb amptutations in two children. Of the six group A streptococcal isolates three produced exotoxins A and B, one produced B and C, one A only, and one B only. Exotoxin production was shown in $\mathbf{4 0}$ of $\mathbf{5 8}$ strains isolated from people outside hospital with sore throats but none of those produced toxin $\mathrm{A}$.

It seems that the marked decline in severe scarlet fever which occurred some $\mathbf{4 0}$ or so years ago was a result of the loss of the organism's ability to produce toxin A but it appears to have relearned the trick. We'd better watch out. 